\title{
A Data Repository for the EDM community:
}

\section{The PSLC DataShop}

\author{
Kenneth R. Koedinger ${ }^{1}$, Ryan S.J.d. Baker ${ }^{2}$, Kyle Cunningham ${ }^{1}$, Alida Skogsholm ${ }^{1}$, \\ Brett Leber ${ }^{1}$, John Stamper ${ }^{1}$ \\ \{krk, kcunning, alida, bleber, jstamper\}@ cs.cmu.edu, rsbaker@wpi.edu \\ ${ }^{1}$ Human-Computer Interaction Institute, Carnegie Mellon University \\ ${ }^{2}$ Department of Social Science and Policy Studies, Worcester Polytechnic Institute
}

\section{Introduction}

In recent years, educational data mining has emerged as a burgeoning new area for scientific investigation. One reason for the emerging excitement about educational data mining is the increasing availability of fine-grained, extensive, and longitudinal data on student learning. These data come from many sources, including standardized tests combined with student demographic data (for instance, www.icpsr.umich.edu/IAED), and videos of classroom interactions [22]. Extensive new data sources have been transformational in science [5] and business (being a major part of the success of key businesses such as Google, FedEx, and WalMart).

In this paper, we present an open data repository of learning data - the Pittsburgh Science of Learning Center DataShop (http://pslcdatashop.org) - which we have designed to have characteristics that make it particularly useful for educational data mining. We discuss the ways in which members of the EDM community are currently utilizing this resource, and how DataShop’s tools support both exploratory data analysis and educational data mining. 
At present, DataShop specializes in data on the interaction between students and educational software, including data from online courses, intelligent tutoring systems, virtual labs, online assessment systems, collaborative learning environments, and simulations. Historically, educational data of this nature have been stored in a wide variety of formats, including streamed log files directly from web-based or non-web-based educational software, summary log files (sometimes including outputs from student models), and researcher-specific database formats (both flat and relational). Moving toward a common set of standards for sharing data, student models, and the results of EDM analyses - key goals of the DataShop project - will facilitate more efficient, extensive storage and use of such data, and more effective collaboration within the community.

DataShop contains data with three attributes that make it particularly useful for educational data mining analyses. First, the data is fine-grained, at the grain-size of semantically meaningful “transactions" between the student and the software, including both the student's action, and the software's response. Second, the data is longitudinal, involving student behavior and learning, in many cases, over the span of an entire semester or year of study. Third, the data is extensive, involving millions of transactions for some of the educational software packages for which DataShop has data. These three characteristics have made the PSLC DataShop useful to many educational data miners, both involved with the PSLC and external to it. We have the ambition of becoming the key venue for sharing educational interaction data and collaborating on its progressive analysis to support scientific discovery in education.

\section{The Pittsburgh Science of Learning Center DataShop}

DataShop is a data repository and web application for learning science researchers and 
educational data mining researchers. It provides secure data storage as well as an array of analysis and visualization tools available through a web-based interface. Data is collected from the PSLC’s six ongoing courses: Algebra (intelligent tutors and collaborative learning), Chemistry (virtual labs, on-line course interactions, and intelligent tutors), Chinese (on-line course interactions and optimized drill), English (intelligent tutors and optimized drill), Geometry (intelligent tutors) and Physics (intelligent tutors and collaborative learning). There are also sources external to the PSLC that regularly contribute data to DataShop, such as middle school math data from the ASSISTments project (http://www.assistment.org) [18] and college on-line course data (e.g., in Statistics) from the Open Learning Initiative (http://cmu.edu/oli). Many other studies and researchers also use DataShop to analyze their data.

DataShop can store a wide variety of types of data associated with a computerized course or study. This includes student-software interaction data (which is capable of being analyzed through the analysis and visualization tools) as well as any related publications, files, presentations, or electronic artifacts a researcher would like to store. In many cases, pre and post tests, questionnaire responses, system screen shots, and demographic data are associated with student interaction data. Mappings between problem steps and knowledge components (either skills or concepts) can be created by researchers and statistically compared to one another.

Courses and studies are represented as datasets, which are organized by project, when relevant. For example, one dataset that has been used in several educational data mining analyses [cf. 6, 7, $9,10,23,24]$ is the "Algebra $12005-2006$ " dataset, which is grouped with similar datasets under the “Algebra Course” project.

The amount of data in DataShop is constantly growing. As of June 2009, DataShop offers 164 
datasets under 50 projects. Across data sets, there are 25 million software-student transactions, representing over 111,000 student hours.

Researchers have utilized DataShop to explore learning issues in a variety of educational domains. These include, but are not limited to, collaborative problem solving in Algebra [31], self-explanation in Physics [20], the effectiveness of worked examples and polite language in a Stoichiometry tutor [25] and the optimization of knowledge component learning in Chinese [28].

\section{Logging and Storage Methods}

Software-student interaction data is typically parsed from messages logged by educational software - such as the intelligent tutor shown in Figure 1 - into the DataShop XML format. While the student learns from the software, the student's actions and the tutor's responses are stored in a log database or file, which is imported into DataShop for storage and analysis.
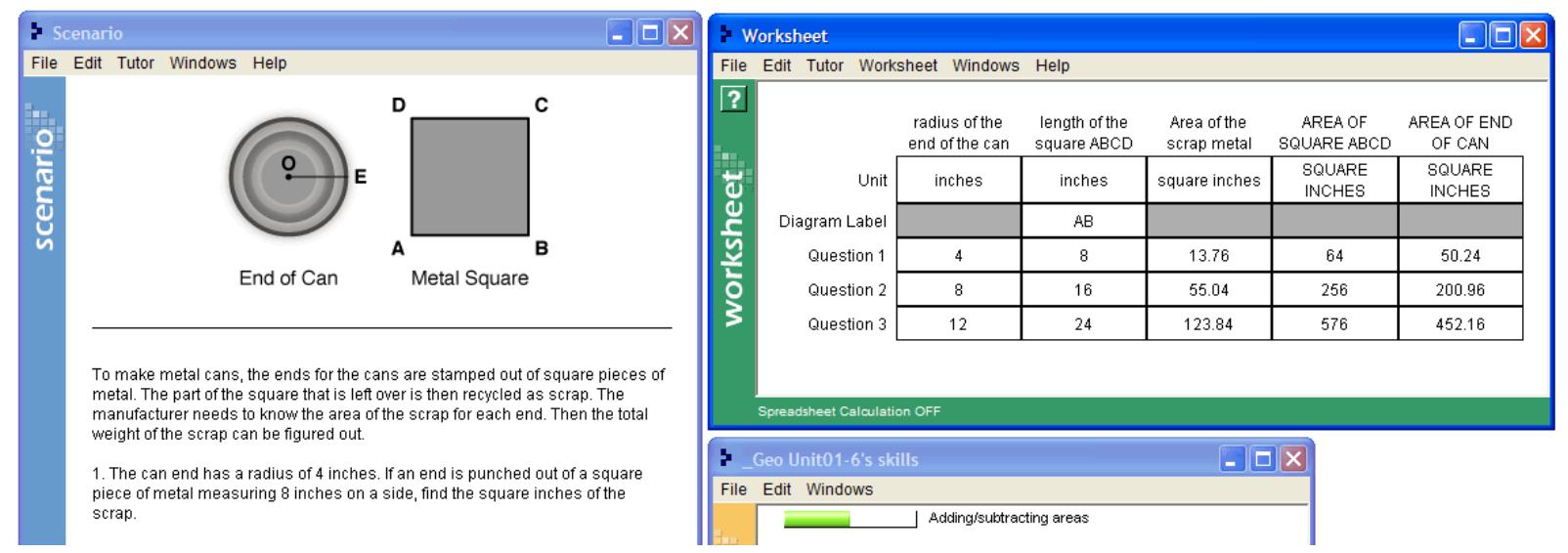

Figure 1. A problem from Carnegie Learning’s Cognitive Tutor Geometry (2005 version).

The DataShop logging format differs from many other educational data formatting standards in that it attempts to capture student-computer interaction history at a fine-grained level, while also 
providing data on the interactions' context. The format does not attempt to describe, a priori, learning resources and how they're transferred [cf. 1, 16] or test content [cf. 32]. In this way, the format is essentially descriptive, not prescriptive. The DataShop logging model is represented by the following constructs [cf. 31]:

- Context message: the student, problem, and session with the tutor

- Tool message: represents an action in the tool performed by a student or tutor

- Tutor message: represents a tutor's response to a student action

Below we see example context, tool, and tutor messages in the DataShop XML format:

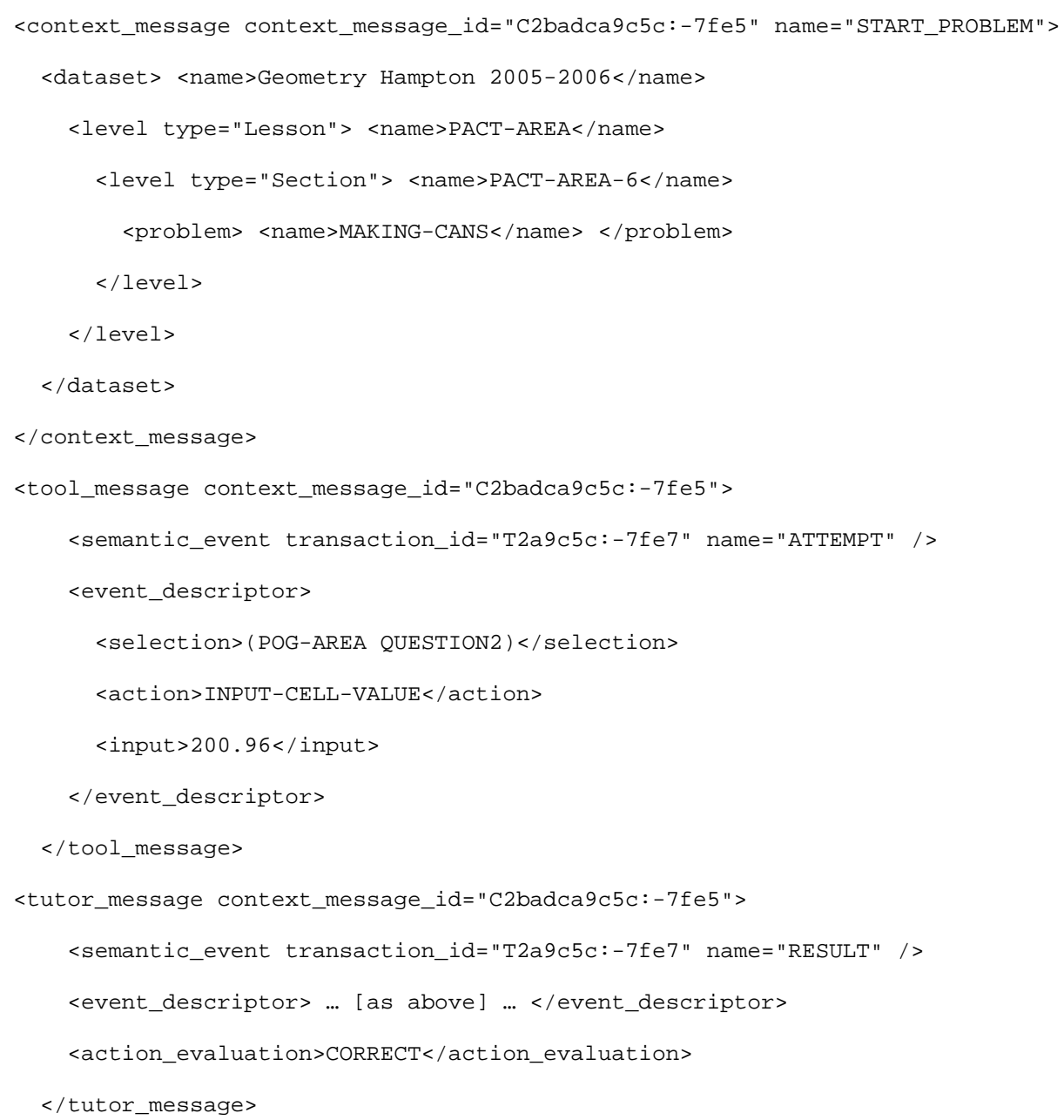


In this example, the student attempted problem "MAKING-CANS" in the "PACT-AREA" lesson of the Geometry tutor, shown in Figure 1. Looking at the tool and tutor message pair, we see the student correctly entered "200.96" as the answer. Tool and tutor messages are paired with each other (by the context message), allowing DataShop to interpret the student action and the tutor's response in conjunction. These pairs are then stored as a single tutor transaction in the database. Table 1 below illustrates how actions from the Making Cans example are interpreted and stored as tutor transactions.

A tutor transaction stores details such as the student identifier, session, time, problem name, problem step (or subgoal) identifier, attempt number, transaction type (e.g., attempt or hint request), student input, tutor response, hint number (if this transaction was a hint request) conditions assigned to the problem step, as well as the knowledge components (skills or concepts) relevant to the transaction. DataShop can also store "untutored" student actions, which include a student clicking "play" on an audio or video segment, navigating through pages of on-line course content, or taking an action in an un-tutored virtual lab like pouring the contents of one virtual beaker into another. These actions are logged as unpaired tool messages (i.e., there is no corresponding tutor message) and are stored in the repository as well. 
Table 1. A simplified tutor transaction excerpt from the "Making Cans” example.

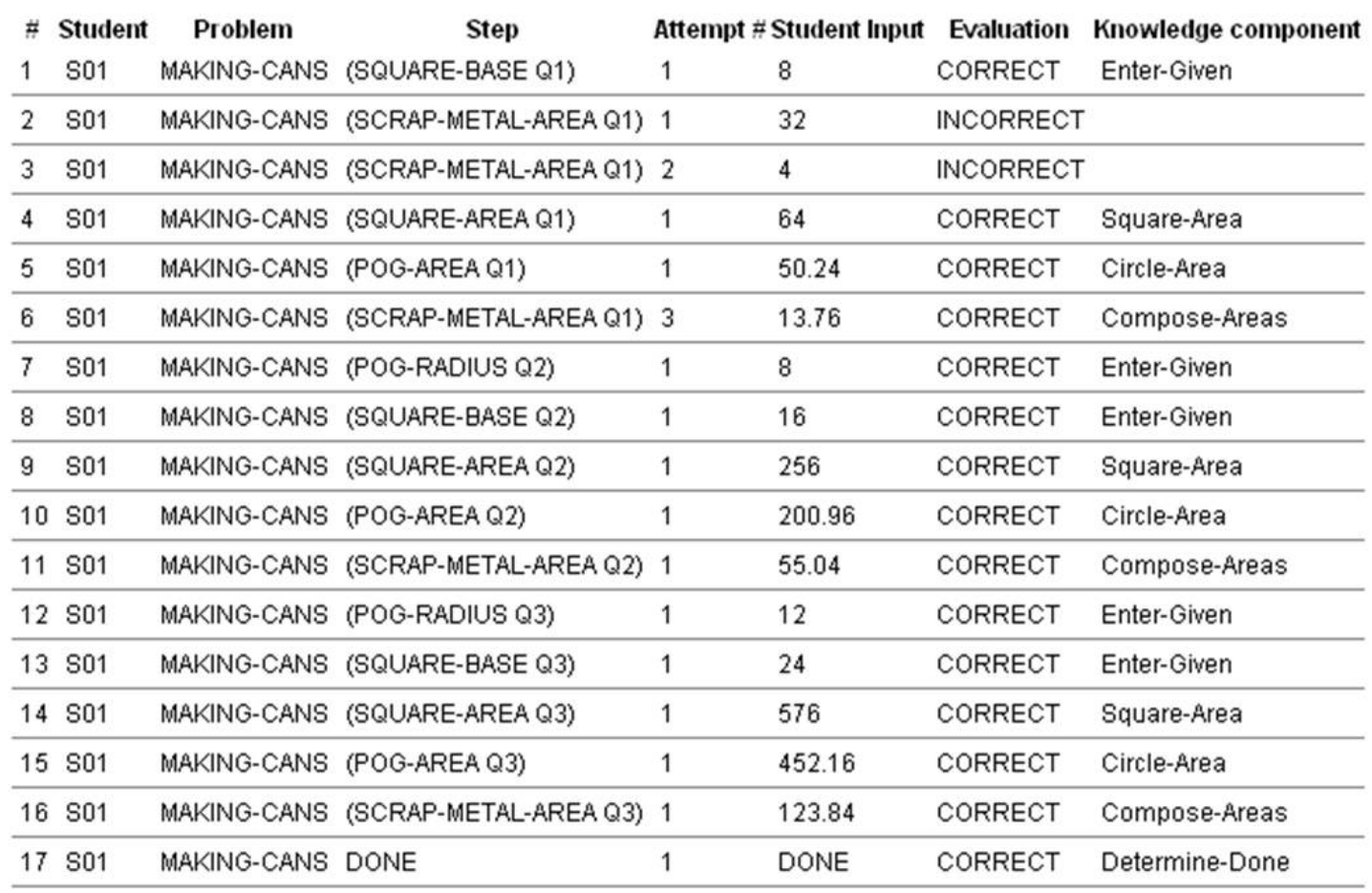

Multiple tool and tutor messages are typically logged for a single problem-solving activity.

Problem-solving activity is broken down into “steps” which represent completion of possible subgoals or pieces of a problem solution. Students often make multiple attempts at a step or get instructional help on a step and each of these attempts or help requests are stored as a separate tutor transaction in the database. In the "Making Cans" example, we see the student attempted the “(SCRAP-METAL-AREA Q1)” step three times (transaction numbers 2, 3 and 6 in Table 1). We can ascertain from the transactions that the student was unsuccessful in her first two attempts, providing an answer of “32” and “4”, both labeled as incorrect by the tutor. On the third attempt, the student successfully completed the problem step, providing an input of " 13.76 ” (as can be seen in Figure 1).

To allow for fast and easy analysis of data and distillation into visualizations (discussed later in 
this chapter), tutor transactions are aggregated into a student-step rollup table. This

“denormalized” table aggregates the data into a record of each step (of each problem) executed

by each student. This table is used by many of the DataShop tools, such as the Performance

Profiler and Learning Curve. An example of how the "Making Cans” tutor transactions are aggregated by student-step is depicted in Table 2 .

Table 2. Data from the “Making Cans” example, aggregated by student-step Step table excerpt

\begin{tabular}{|c|c|c|c|c|c|c|c|c|c|}
\hline$\#$ & Student & Problem & Step & Opportunity Count & Total Incorrects & \multicolumn{4}{|c|}{ Total Hints Assistance Score Error Rate Knowledge component } \\
\hline 1 & S01 & WATERING_VEGGIES & (WATERED-AREA Q1) & 1 & 0 & 0 & 0 & 0 & Circle-Area \\
\hline 2 & S01 & WATERING_VEGGIES & (TOTAL-GARDEN Q1) & 1 & 2 & 1 & 3 & 1 & Rectangle-Area \\
\hline 3 & $\mathrm{~S} 01$ & WATERING_VEGGIES & (UNWATERED-AREA Q1) & 1 & 0 & 0 & 0 & 0 & Compose-Areas \\
\hline 4 & S01 & WATERING_VEGGIES & DONE & 1 & 0 & 0 & 0 & 0 & Determine-Done \\
\hline 5 & S01 & MAKING-CANS & (POG-RADIUS Q1) & 1 & 0 & 0 & 0 & 0 & Enter-Given \\
\hline 6 & S01 & MAKING-CANS & (SQUARE-BASE Q1) & 1 & 0 & 0 & 0 & 0 & Enter-Given \\
\hline 7 & S01 & MAKING-CANS & (SQUARE-AREA Q1) & 1 & 0 & 0 & 0 & 0 & Square-Area \\
\hline 8 & S01 & MAKING-CANS & (POG-AREA Q1) & 2 & 0 & 0 & 0 & 0 & Circle-Area \\
\hline 9 & S01 & MAKING-CANS & (SCRAP-METAL-AREA Q1) & 2 & 2 & 0 & 2 & 1 & Compose-Areas \\
\hline 10 & So1 & MAKING-CANS & (POG-RADIUS Q2) & 2 & 0 & 0 & 0 & 0 & Enter-Given \\
\hline 11 & S01 & MAKING-CANS & (SQUARE-BASE Q2) & 2 & 0 & 0 & 0 & 0 & Enter-Given \\
\hline 12 & $\mathrm{~S} 01$ & MAKING-CANS & (SQUARE-AREA Q2) & 2 & 0 & 0 & 0 & 0 & Square-Area \\
\hline 13 & S01 & MAKING-CANS & (POG-AREA Q2) & 3 & 0 & 0 & 0 & 0 & Circle-Area \\
\hline 14 & $S 01$ & MAKING-CANS & (SCRAP-METAL-AREA Q2) & 3 & 0 & 0 & 0 & 0 & Compose-Areas \\
\hline 15 & S01 & MAKING-CANS & (POG-RADIUS Q3) & 3 & 0 & 0 & 0 & 0 & Enter-Given \\
\hline 16 & S01 & MAKING-CANS & (SQUARE-BASE Q3) & 3 & 0 & 0 & 0 & 0 & Enter-Given \\
\hline 17 & S01 & MAKING-CANS & (SQUARE-AREA Q3) & 3 & 0 & 0 & 0 & 0 & Square-Area \\
\hline 18 & $\mathrm{~S} 01$ & MAKING-CANS & (POG-AREA Q3) & 4 & 0 & 0 & 0 & 0 & Circle-Area \\
\hline 19 & So1 & MAKING-CANS & (SCRAP-METAL-AREA Q3) & 4 & 0 & 0 & 0 & 0 & Compose-Areas \\
\hline 20 & $S 01$ & MAKING-CANS & DONE & 2 & 0 & 0 & 0 & 0 & Determine-Done \\
\hline
\end{tabular}

One other key type of information stored in DataShop is information on the "knowledge components” involved in steps. Each step in a problem requires the student to know somethinga relevant concept or skill — to perform the step correctly. This small unit of knowledge is termed a "knowledge component” (see http://www.learnlab.org/research/wiki for more detail on this construct). In the "Making Cans” example, we see the knowledge component "ComposeAreas” assigned to the correct transaction (row 6 of Table 1) for the "(SCRAP-METAL-AREA Q1)” step. A knowledge component codes for a general student capability to accomplish steps in tasks. Knowledge component modeling, the process of assigning knowledge components to 
steps, bolsters the usefulness of intelligent tutor data, increasing the feasibility of assessing a student's knowledge relevant to a given step [cf. 7, 8]. A step can have zero, one, or multiple knowledge components associated with it.

To document this required concept or skill, a tutor author can label steps with the hypothesized knowledge component(s) required for correct completion of the step, and this encoding can be imported into DataShop along with the data. Alternatively, researchers can develop their own knowledge component to step mappings, either through knowledge engineering, exploratory data analysis (using DataShop’s Performance Profiler - more on this later), or educational data mining [cf. 14], and import these mappings into DataShop through the web application. DataShop provides researchers with an assessment of the goodness of each model (e.g., the Bayesian Information Criterion [cf. 30]), which can be used to determine which model best represents the data. Some data sets now have as many as ten different knowledge component models associated with them, by researchers at multiple universities (for instance, the "Geometry Area (1996-1997)” data set).

\section{Importing and Exporting Learning Data}

Data may be imported into the DataShop repository through XML or a tab-delimited text file format. Logging to DataShop XML provides the richest and most complete data. If logging via XML, educational software can send messages directly to the DataShop logging server in real time. This approach is used by example-tracing tutors built in the CTAT framework (ctat.pact.cs.cmu.edu) [cf. 3]. Logs are automatically processed on a nightly basis, making them available for analysis or export through the web application. Alternatively, a computer tutor can write XML to files on the local hard disk (for example, if a tutor is running off-line) and then 
send the data to the logging server at a later time. Data in a pre-existing log format can also be converted to DataShop XML and then imported into the repository. This procedure has worked well for data collected by several types of educational software including Andes (www.andes.pitt.edu) [19], mathematics Cognitive Tutors (carnegielearning.com) [4], REAP (reap.cs.cmu.edu) [13], Optimized vocabulary practice [28], and Assistments (assistments.org) [18]. The tab-delimited format of a transaction table can alternatively be used to import from a preexisting source.

DataShop offers various data export options through the web application, each delivered in a tabdelimited text file. These include transaction and student-step level exports (as illustrated in Tables 1 \& 2), and a student-problem aggregate export.

\section{Analysis and Visualization Tools}

The DataShop web application provides several tools to assist with analyzing and visualizing repository data. These tools can be used in conjunction to jump-start data analysis: A researcher can determine if students are learning by viewing learning curves, then drill down on individual problems, knowledge components, and students to analyze performance in greater detail.

The following DataShop tools are available for exploratory data analysis:

- $\quad$ Dataset Info: provides dataset metrics, contextual information, demographics, high-level descriptive information (number of students, transactions, knowledge components, etc.) as well as papers, files, a problem summary table, and the ability to export and import knowledge component models. 
- Error Report: presents each student's first attempt at a problem or knowledge component, including if he or she was correct, the number of students or observations, and the details of the student's answer.

- $\quad$ Performance Profiler: multi-purpose tool that visualizes student performance at various grain sizes (problem, step, curriculum level, knowledge component, and student) and offers a choice of measures of performance (error rate, assistance score, average number of incorrect actions, average number of hints, and residual error rate). There is support for selecting subsets of interest (“samples”) among the problems or knowledge components in a curriculum. Figure 2 below shows student performance (proportion of correct answers, errors, and hint requests) on a sub-set of the problems in Cognitive Tutor Algebra; the y-axis ("Problem”) represents individual problems, and the proportion of each performance category is shown along the x-axis.

Dataset Info $\quad$ Performance Profiler Error Report $\quad$ Learning Curve Export

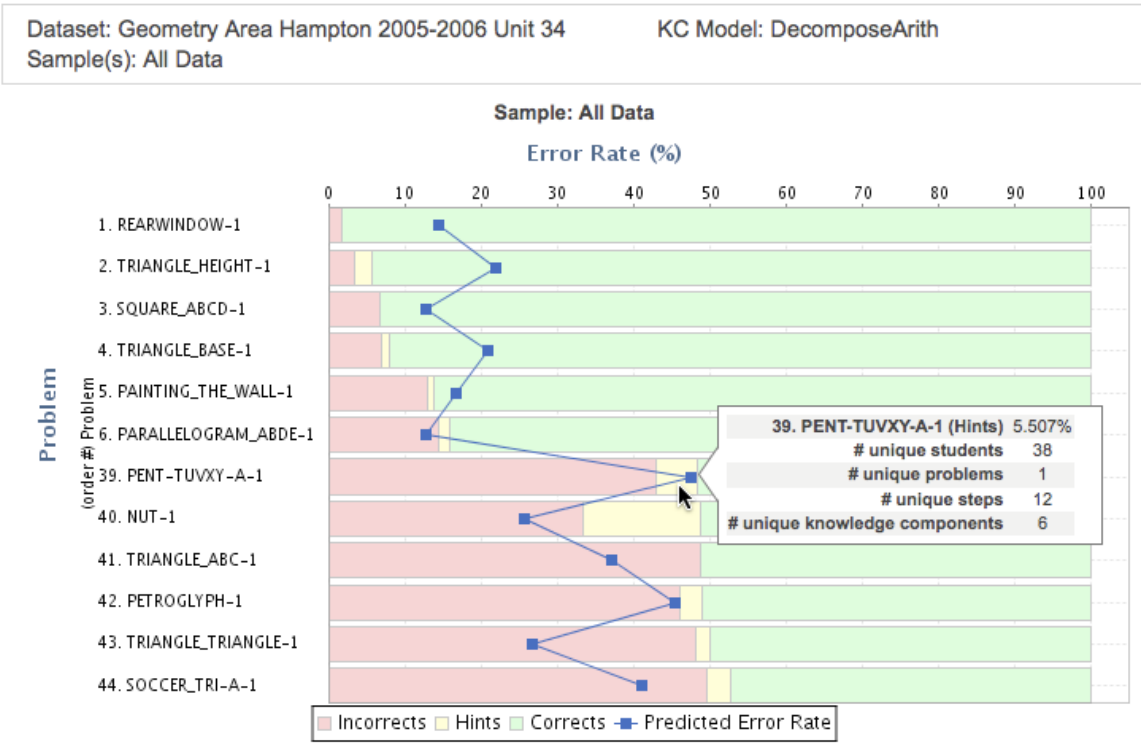

Figure 2. Performance Profiler tool showing the average Error Rate, which is the incorrect entries (red) plus hints (yellow) on students' first attempt at each step across a selection of Problems from a Geometry Area data set. Using controls not pictured, the user has selected to view the 6 problems with the lowest 
error rate and the 6 with the highest error rate. The blue points are predictions based a particular knowledge component model and the statistical model behind the Learning Factors Analysis [14] algorithm.

- Learning Curve: visualizes student learning changes over time. The tool can visualize the change over time in error rate, assistance score, correct step time, and other metrics as well. The Learning Factors Analysis model [14] can provide predicted values for error rate learning curves. Figure 3 below depicts error rate learning curves generated by DataShop. In this graph, "error rate", or the percentage of students that asked for a hint or made an incorrect attempt on their first attempt on steps associated with a specific knowledge component, is shown on the y-axis. The x-axis (“Opportunity”) indicates the nth time (e.g., 4 is the $4^{\text {th }}$ time) a student has (according to the current model) had an opportunity to use a knowledge component to solve a step in a problem. Each unique step in a problem is distinct from other problem-solving steps, even if they involve the same knowledge component(s).
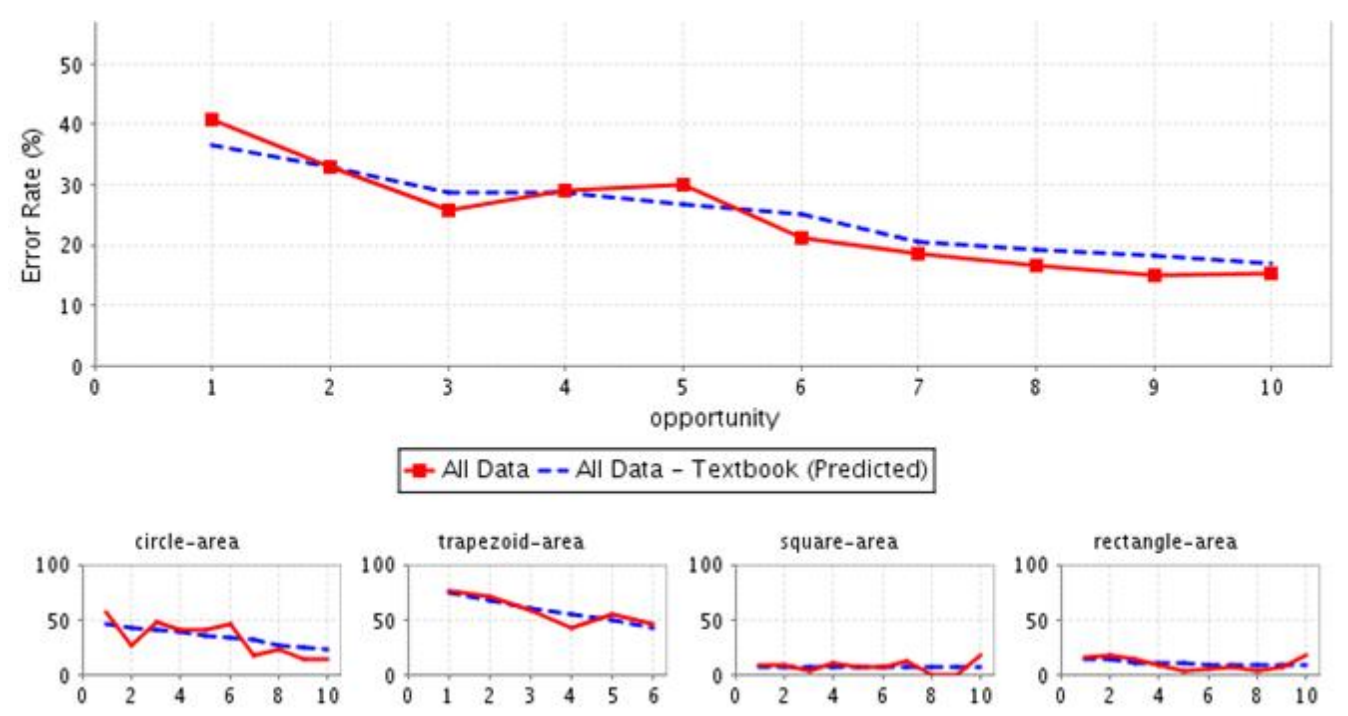

Figure 3. Error Rate Learning Curve with predicted values from a Geometry Area dataset. The solid curve represents the actual values, each point is an average across all students and knowledge components for the given opportunity. The dashed curve represents the predicted curve values, based on the Learning Factor Analysis (LFA) model [14], a variant of Item Response Theory. 


\section{Uses of the PSLC DataShop}

As indicated above, many recent analyses of data from DataShop have been performed in a variety of domains. A number of other studies have used, tested or extended the analysis techniques employed in DataShop including investigations in reading [21], Physics [26], and Geometry [29]. Often analyses have been targeted at finding ways to improve student learning. In some cases, the work has been taken full circle such that an analysis led to an instructional redesign that was demonstrated to improve student learning beyond that realized by the original instruction. We provide a couple of examples.

Cen, Junker, and Koedinger performed a learning curve analysis using the Learning Factors Analysis (LFA) algorithm based on data from the Area unit of the Geometry Cognitive Tutor [15]. They noticed that while students were required to over-practice some easy target knowledge components or skills (see square-area in Figure 2), they under-practiced some harder skills (see trapezoid-area in Figure 2). Based on observation and further analysis, they created a new version of the geometry tutor by resetting parameters that determine how often skills are practiced. They ran a classroom experiment where students in a course were pre- and post-tested and randomly assigned to use either the previous or the new tutor version. Students using the new version took $20 \%$ less time to finish the same curriculum units (because over-practice was eliminated) and learned just as much as measured by normal, transfer, and long-term retention tests.

A second demonstration of a data mining project that "closed the loop" is work by Baker et al. [8] who had done formal observations of student behavior in computer labs while working 
through lessons of a middle school math Cognitive Tutor. Among a number of categories of disengaged behavior, he found that "gaming the system" had the largest correlation with poor learning outcomes. Gaming refers to student behavior that appears to avoid thinking and learning through systematic guessing or fast and repeated requests for increasing help. Baker used machine learning techniques to build a "detector" capable of processing student log information, in real time, to determine when students were gaming. The detector became the basis for an intervention system, a "meta tutor", designed to discourage gaming and engage students in supplementary instruction on topics they had gamed. A controlled experiment demonstrated student-learning benefits associated with this adaptive selection of supplementary instruction for students observed to be gaming. Since then, the gaming detector has been used within analyses of why students game [11], and precisely how gaming leads to poorer learning.

Broadly, the data available in DataShop is driving the development of more precise computational models of human cognition, motivation, and learning. In particular, an ongoing area of research using DataShop data is the empirical evaluation and improvement of knowledge representations [cf. 12, 17, 33]. As noted in a major national report, "psychometric validation of [on-line] assessments is needed so they can be compared with conventional assessments, and complement and ultimately supplant them” [2].

\section{Data Annotation: A Key Upcoming Feature}

Over the next year, one of our key development goals is to make it possible for researchers to easily upload the results of data mining and computational modeling projects to the data sets where those analyses took place, annotating transactions, problem steps, and students with reference to any cognitive, meta-cognitive, or motivational constructs that can be expressed at 
these grain-sizes. Functionality will be added to enable researchers to connect to DataShop either programmatically (via a web service) or by uploading annotations within the web application (as is currently possible for knowledge component models). We believe that adding this capacity to DataShop will increase its utility, as today’s analyses often build on yesterday’s analyses. For instance, models of gaming the system and off-task behavior have relied upon estimates of the probability that the student knew the relevant knowledge components at the time of the behavior. It will also allow for a larger number of competitive analyses [cf. 6], where multiple modeling methods are applied to the same data set, and evaluated for comparative goodness of fit.

\section{Conclusions}

We have described PSLC’s DataShop, an open repository and web-based tool suite for storing and analyzing click-stream data, fine-grained longitudinal data generated by online courses, assessments, intelligent tutoring systems, virtual labs, simulations, and other forms of educational technology. In contrast to other types of educational data such as video and schoollevel data, data in DataShop includes a rich set of semantic codes that facilitate automated analysis and meaningful interpretation.

The PSLC DataShop uniform data format is an initial attempt to develop a common standard that we hope will be useful to the field if not as is, then in driving better or more useful common standards. In addition to being a source for learning data, it is also a place where researchers can deposit data and then get help from other researchers who can perform secondary analysis on this data.

DataShop allows free access to a wide variety of data sets and analysis tools. These tools help 
researchers visualize student performance, difficulties, and learning over time. Such analyses can lead to demonstrably better instructional designs. The data can also drive improved models of student cognition, affect, and learning that can be used to improve on-line assessment and online learning. We take as a premise that the human brain constructs knowledge based on a variety of input sources (e.g., verbal, visual, physical) and in a fashion and at a grain size that may or may not conform to the structure as conceived by an instructor or domain expert. The question of how the latent nature and content of human knowledge representation can be discovered from data is a deep and important scientific question, like for instance, the nature of the human genome. To answer this question requires a vast collection of relevant data, associated analysis methods, and new theory.

\section{Acknowledgements}

Research supported by National Science Foundation award number SBE-0354420 for the Pittsburgh Science of Learning Center.

\section{References}

1. Advanced Distributed Learning. 2003. SCORM Overview. Unpublished white paper. Alexandria, VA: Advanced Distributed Learning.

2. Ainsworth, S., Honey, M., Johnson, W. L., Koedinger, K. R., Muramatsu, B., Pea, R., Recker, M., and Weimar, S. Cyberinfrastructure for Education and Learning for the Future (CELF): A Vision and Research Agenda. 2005. Washington, DC: Computing Research Association. 
3. Aleven, V., McLaren, B., Sewall, J. and Koedinger, K. 2006. The Cognitive Tutor Authoring Tools (CTAT): Preliminary Evaluation of Efficiency Gains. Proceedings of the 8th International Conference on Intelligent Tutoring Systems: 61-70.

4. Anderson, J. R., Corbett, A. T., Koedinger, K. R., and Pelletier, R. 1995. Cognitive tutors: Lessons learned. The Journal of the Learning Sciences 4 (2): 167-207.

5. Atkins, D.E. (Ed.). 2003. Revolutionizing Science and Engineering Through Cyberinfrastructure: Report on the National Science Foundation Blue-Ribbon Advisory Panel on Cyberinfrastructure. Arlington, Virginia: National Science Foundation. http://www.cise.nsf.gov/sci/reports/atkins.pdf

6. Baker, R.S.J.d., Corbett, A.T., Aleven, V. 2008. Improving Contextual Models of Guessing and Slipping with a Truncated Training Set. Proceedings of the 1st International Conference on Educational Data Mining: 67-76.

7. Baker, R.S.J.d., Corbett, A.T., Aleven, V. 2008. More Accurate Student Modeling Through Contextual Estimation of Slip and Guess Probabilities in Bayesian Knowledge Tracing. Proceedings of the 9th International Conference on Intelligent Tutoring Systems: 406-415.

8. Baker, R., Corbett, A., Koedinger, K. R., Evenson, S., Roll, I., Wagner, A., Naim, M., Raspat, J., Baker, D., and Beck, J. 2006. Adapting to when students game an intelligent tutoring system. In M. Ikeda, K. D. Ashley, T.-W. Chan (Eds.) Proceedings of the 8th International Conference on Intelligent Tutoring Systems: 392-401.

9. Baker, R.S.J.d., de Carvalho, A. M. J. A. 2008. Labeling Student Behavior Faster and More Precisely with Text Replays. Proceedings of the 1st International Conference on Educational Data Mining: 38-47. 
10. Baker, R.S.J.d., de Carvalho, A.M.J.A., Raspat, J., Aleven, V., Corbett, A.T., Koedinger, K.R. 2009. Educational Software Features that Encourage and Discourage "Gaming the System". Proceedings of the 14th International Conference on Artificial Intelligence in Education: 475-482.

11. Baker, R., Walonoski, J., Heffernan, N., Roll, I., Corbett, A., Koedinger, K. 2008. Why Students Engage in "Gaming the System" Behavior in Interactive Learning Environments. Journal of Interactive Learning Research 19 (2): 185-224.

12. Barnes, T., D. Bitzer, and Vouk, M. 2005. Experimental analysis of the q-matrix method in knowledge discovery. Proceedings of the 15th International Symposium on Methodologies for Intelligent Systems, May 25-28, 2005, Saratoga Springs, NY.

13. Brown, J., Frishkoff, G., Eskenazi, M. 2005. Automatic Question Generation for Vocabulary Assessment. Proceedings of the Annual Human Language Technology Meeting: 249-254.

14. Cen, H., Koedinger, K., and Junker, B. 2006. Learning Factors Analysis - A general method for cognitive model evaluation and improvement. Proceedings of the 8th International Conference on Intelligent Tutoring Systems.

15. Cen, H., Koedinger, K., and Junker, B., 2007. Is over practice necessary? - Improving learning efficiency with the cognitive tutor through educational data mining. In Rose Luckin and Ken Koedinger (Eds.) Proceedings of the 13th International Conference on Artificial Intelligence in Education: 511-518.

16. Duval, E., and Hodgins, W. 2003. A LOM Research Agenda. Proceedings of WWW2003 Twelfth International World Wide Web Conference, 20-24 May 2003, Budapest, Hungary.

17. Falmagne, J.-C., Koppen, M., Villano, M., Doignon, J.-P. 1990. Introduction to knowledge spaces: How to build, test, and search them. Psychological Review 97: 201-224. 
18. Feng, M., Heffernan, N.T. 2007. Towards Live Informing and Automatic Analyzing of Student Learning: Reporting in ASSISTment System. Journal of Interactive Learning Research 18 (2): 207-230.

19. Gertner, A.S., and VanLehn, K. 2000. Andes: A Coached Problem-Solving Environment for Physics. Proceedings of the $5^{\text {th }}$ International Conference on Intelligent Tutoring Systems: 133-142.

20. Hausmann, R., and VanLehn, K. 2007. Self-explaining in the classroom: Learning curve evidence. In McNamara and Trafton (Eds.), Proceedings of the 29th Annual Cognitive Science Society: 1067-1072.

21. Leszczenski, J. M. and Beck J. E. 2007. What's in a word? Extending learning factors analysis to model reading transfer. Proceedings of the Educational Data Mining Workshop at the 14th International Conference on Artificial Intelligence in Education: 31-39.

22. MacWhinney, B., Bird, S., Cieri, C., Martell, C. 2004. TalkBank: Building an open unified multimodal database of communicative interaction. Proceedings of the $4^{\text {th }}$ International Conference on Language Resources and Evaluation.

23. Matsuda, N., Cohen, W., Sewall, J., Lacerda, G., and Koedinger, K. R. 2007. Evaluating a simulated student using real students data for training and testing. In C.Conati, K. McCoy, and G. Paliouras, (Eds.) Proceedings of the 11th International Conference on User Modeling, UM2007: 107-116.

24. Matsuda, N., Cohen, W., Sewall, J., Lacerda, G., and Koedinger, K. R. 2007. Predicting students’ performance with SimStudent: learning cognitive skills from observation. In R. Lukin, K.R. Koedinger, and J. Greer, (Eds.) Proceedings of the 13th International Conference on Artificial Intelligence in Education: 467-476. 
25. McLaren, B. M., Lim, S., Yaron, D., and Koedinger, K. R. 2007. Can a polite intelligent tutoring system lead to improved learning outside of the lab? In Luckin and Koedinger (Eds.) Proceedings of the 13th International Conference on Artificial Intelligence in Education: 433-440.

26. Nwaigwe, A., Koedinger, K.R., VanLehn, K., Hausmann, R., and Weinstein, A. 2007. Exploring alternative methods for error attribution in learning curves analyses in intelligent tutoring systems. In Luckin and Koedinger (Eds.) Proceedings of the 13th International Conference on Artificial Intelligence in Education: 246-253.

27. Ohlsson, S. and Mitrovic, A. 2006. Constraint-based knowledge representation for individualized instruction. Computer Science and Information Systems: 1-22.

28. Pavlik Jr., P. I., Presson, N., and Koedinger, K. R. 2007. Optimizing knowledge component learning using a dynamic structural model of practice. In R. Lewis and T. Polk (Eds.) Proceedings of the Eighth International Conference of Cognitive Modeling.

29. Rafferty, A. N. and Yudelson, M. 2007. Applying learning factors analysis to build stereotypic student models. Proceedings of 13th International Conference on Artificial Intelligence in Education.

30. Raftery, A. 1995. Bayesian Model Selection in Social Science Research. Sociological Methodology 28: 111-163.

31. Ritter, S., and Koedinger, K. R. 1998. An architecture for plug-in tutor agents. Journal of Artificial Intelligence in Education 7 (3-4): 315-347.

32. Smythe, C., and Roberts, P. 2000. An Overview of the IMS Question \& Test Interoperability Specification, Proceedings of the Conference on Computer Aided Assessment (CAA'2000), Leicestershire, UK. 
33. Tatsuoka, K. 1983. Rule space: An approach for dealing with misconceptions based on item response theory. Journal of Educational Measurement 20 (4): 345-354. 